\title{
openheart Association between adiposity and systemic atherosclerosis: a protocol of a cross-sectional autopsy study
}

\author{
Aline Nishizawa, ${ }^{1,2}$ Claudia Kimie Suemoto, ${ }^{1,2,3}$ Daniela Souza Farias,, ${ }^{1,2}$ \\ Fernanda Marinho Campos, ${ }^{1}$ Karen Cristina Souza da Silva, ${ }^{1,2}$ \\ Anderson Cuelho, ${ }^{1,2}$ Renata Elaine Paraízo Leite, ${ }^{2,3}$ Renata Eloah de \\ Lucena Ferretti-Rebustini, ${ }^{2,4}$ Lea Tenenholz Grinberg, ${ }^{2,5}$ José Marcelo Farfel, ${ }^{2,3}$ \\ Wilson Jacob-Filho, ${ }^{2,3}$ Carlos Augusto Pasqualucci ${ }^{1,2,6}$
}

To cite: Nishizawa A,

Suemoto CK, Farias DS, et al. Association between adiposity and systemic atherosclerosis: a protocol of a cross-sectional autopsy study. Open Heart 2016;3: e000433. doi:10.1136/ openhrt-2016-000433

Received 14 March 2016 Revised 13 May 2016 Accepted 30 June 2016

\section{CrossMark}

For numbered affiliations see end of article.

Correspondence to Professor Claudia Kimie Suemoto, Discipline of Geriatrics, University of Sao Paulo Medical School, Dr Arnaldo Av., 455, room 1353, Sao Paulo 01246-903, SP, Brazil; cksuemoto@usp.br

\section{ABSTRACT}

Introduction: Adiposity has been associated with atherosclerosis in clinical studies. However, few autopsy studies have investigated this association, and they had only examined the coronary artery disease. Moreover, most studies had small sample sizes and were limited to middle-aged or young adults. Our aim is to investigate the association between adiposity and systemic atherosclerosis in an autopsy study.

Methods and analysis: A sample of 240 deceased with 30 years or more will be evaluated. The sample size was calculated using the lowest correlation coefficient found in previous studies $(r=0.109)$, assuming a power of $90 \%$ and $\alpha=0.05$. We will collect information about sociodemographics, frequency of previous contact of the deceased's next of kin and cardiovascular risk factors. We will measure neck, waist and hip circumferences, weight, height and abdominal subcutaneous tissue thickness, and then we will calculate the body mass index, waist-to-hip ratio, waist-to-height ratio and body shape index. We will also weigh the pericardial and abdominal visceral fat, the heart, and we will measure the left ventricular wall thickness. We will evaluate the presence of myocardial infarction, the degree of atherosclerosis in the aorta, carotid, coronary and cerebral arteries and plaque composition in carotid, coronary and cerebral arteries. For each individual, we will fix arterial and adipose tissue samples in $10 \%$ formalin and freeze another adipose tissue sample at $-80^{\circ} \mathrm{C}$ for future studies. Ethics and dissemination: Ethical approval was granted by the Ethics Committee of University of Sao Paulo Medical School, Brazil. Results will be submitted for publication in a peer-reviewed journal.

\section{INTRODUCTION}

Ischaemic heart disease and stroke were the leading causes of death worldwide in 2013 and were estimated to be responsible for $14.8 \%$ and $11.7 \%$ of all deaths, respectively. In 2012, 14.1 million people died from these diseases. $^{1}{ }^{2}$ Moreover, ischaemic heart

\section{KEY QUESTIONS}

What is already known about this subject?

- Anthropometric measurements and epicardial and pericardial fat were associated with atherosclerosis in coronary and carotid arteries in previous imaging studies.

- Few autopsy studies investigated the association of atherosclerosis with anthropometric measurements and visceral fat. Moreover, they did not evaluate atherosclerosis at sites other than coronary arteries, had small sample sizes, and were limited to middle-aged or young adults.

What does this study add?

- We will quantify the paracardial and epicardial fat and measure atherosclerosis severity in carotid, aorta, cerebral, and coronary arteries using morphometric methods in an autopsy study with a large number of subjects from different age groups and ethnicities.

- We will also investigate the association of systemic atherosclerosis with a variety of anthropometric measures, like "body shape index", abdominal subcutaneous tissue thickness, body mass index, waist-to-hip ratio, waist-to-height ratio, and neck and abdominal circumference.

How might this impact on clinical practice?

- Identifying which measure is more associated with systemic atherosclerosis will allow to target individuals at higher risk of cardiovascular events.

- This study will provide a diverse tissue collection of arteries and visceral fat, which will be a unique opportunity for future collaborative studies and will contribute to advance the understanding of the relationship between systemic atherosclerosis and adiposity.

disease is the leading cause of DALYs (Disability-Adjusted Life Years) in the world (2342 per 100000 cases), and the stroke is the third leading cause of DALYs (1998 per 
100000 cases). ${ }^{1}$ Therefore, the identification of risk factors $^{3}$ is important in preventing atherosclerosis and decreasing the cardiovascular disease burden.

Measures of adiposity, such as anthropometrics and thoracic and abdominal visceral fat measurements, have been associated with atherosclerosis. ${ }^{4-10}$ However, most of the previous studies have investigated the association between anthropometric and/or visceral fat measurements with systemic atherosclerosis using imaging methods such as ultrasound, ${ }^{81112} \mathrm{CT}^{5},{ }^{910}$ angiography $^{79}$ and echocardiography. ${ }^{7}{ }^{11}$ In a review study of Alexopoulos $e t a l,{ }^{13}$ all studies that investigated the association of visceral adipose tissue with atherosclerosis used imaging methods.

Several disadvantages of these methods are well known, for instance, angiography is an invasive method that exposes the individual to radiation; CT also exposes patients to ionising radiation and the exact anatomical borders of structures in non-contrasted exams are difficult to define using imaging methods, ${ }^{814}$ and paracardial fat is difficult to delimit by echocardiography. ${ }^{15}$ Although postmortem examination provides a more accurate measure of atherosclerosis and visceral fat due to the direct assessment of these structures, few autopsy studies compared atherosclerosis to anthropometric measurements and visceral fat. ${ }^{4}{ }^{16-22}$ In addition, none of these studies evaluated atherosclerosis at sites other than coronary arteries. Most of these studies also had small sample sizes and were limited to middle-aged or young adults.

We aim to investigate the association between adiposity measured by the weight of the visceral fat and anthropometric measurements with morphometric measures of atherosclerosis in the aorta, coronary, carotid and cerebral arteries in a large sample from different age groups.

\section{METHODS AND ANALYSIS Study design}

A cross-sectional observational study is designed.

\section{Study setting}

This study will be conducted at the Sao Paulo Autopsy Service (SPAS), Laboratory of Cardiovascular Pathology and the Pathophysiology in Aging Lab from the Department of Pathology, University of Sao Paulo, Sao Paulo, Brazil.

Since 1931, the SPAS has performed autopsies of individuals who died in the city of Sao Paulo and who have had an unknown natural cause of death. It performs more than 13000 autopsies per year. ${ }^{23}$ In 2004, we started a successful initiative at the SPAS to investigate brain ageing and neurodegenerative diseases. The Brain Bank of the Brazilian Aging Brain Study Group (BB-BABSG) has collected more than 3000 brains over the last decade to provide the materials and data for a number of publications in high-quality international journals. ${ }^{24-26}$ We will extend the work of the BB-BABSG to evaluate the atherosclerosis process.

\section{Study participants}

A convenience sample of 240 deceased individuals referred to the SPAS, and whose next of kin (NOK) agrees to participate will be included in this study.

Inclusion criterion:

A. Individuals aged 30 years or more.

Exclusion criteria (figure 1):

A. The NOK of the deceased provides inconsistent data during the clinical interview.

B. The NOK of the deceased does not have at least weekly contact with the deceased.

C. There are visible evidence of factors modifying anthropometric measures, such as ascites, severe abdominal distension and pregnancy.

D. Loss of $10 \%$ or more of usual weight during the 6 months prior to death.

E. Arteries or visceral fat of the individuals are retained at the autopsy by the pathologist.

F. Postmortem interval (PMI) $>24$ hours.

G. Signs of body autolysis, according to the Crossley criteria. $^{27}$

\section{Outcome measures}

The primary outcome of interest will be atherosclerosis that is measured by the stenosis index in coronary, carotid and cerebral arteries and by the degree of atherosclerosis in the aorta. The secondary outcome will be the number of atherosclerotic plaques in cerebral and coronary arteries. All outcomes will be measured after autopsy.

\section{Data collection}

The NOK of the deceased will answer a semistructured clinical interview that will be administered by trained nurses concerning sociodemographics (age, gender, race, marital status, schooling and socioeconomic status (SES)), frequency of his/her previous contact with the deceased (weekly or daily) and cardiovascular risk factors (hypertension, diabetes mellitus, coronary artery disease (CAD), heart failure, dyslipidaemia, stroke, smoking, alcohol use and physical inactivity). Schooling will be quantified in years of formal education. SES will be evaluated using a Brazilian Scale that considers the quantity of household goods and the education level of the household owner, classifying in eight ordinal categories. ${ }^{28}$ These categories will be grouped to represent the upper, medium and lower social classes. Smoking and alcohol use will be classified in never, current or former. Physical inactivity will be defined as the individual who does not perform any physical activity. Frequency of physical activity per week will be also determined. The main cause of death and the PMI will be obtained from the autopsy report.

\section{Anthropometric and visceral fat measurements}

Anthropometric and visceral fat measurements will be obtained by trained persons following standardised procedures. 


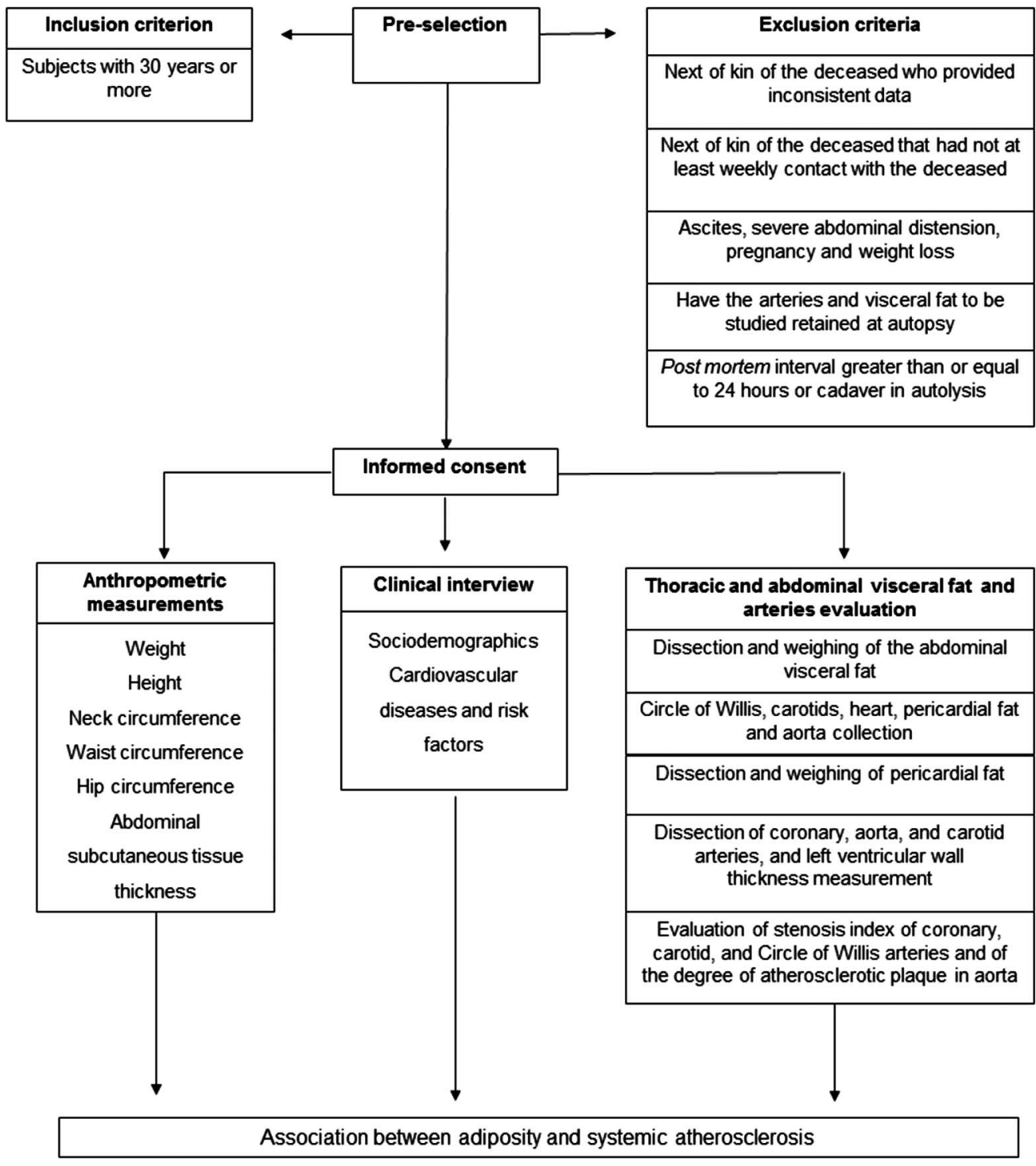

Figure 1 Study outline.

Weight will be measured in kilogram $(\mathrm{kg})$ without clothes in the supine position using a calibrated electronic scale. Height will be measured in centimetre $(\mathrm{cm})$ in the supine position using a stadiometer. After obtaining these measurements, the body mass index (BMI) will be calculated by dividing the weight (in $\mathrm{kg}$ ) by the square of the height (in metres).$^{29}$ Neck circumference (NC) will be measured in the region above the cricoid cartilage. ${ }^{30}$ The waist circumference (WC) measurement will be performed in the region of the umbilicus, and the hip circumference (HC) will be performed in the largest area on the great trochanters. ${ }^{31}$ The abdominal subcutaneous tissue thickness (ASTT) will be measured at the abdominal midline, $4 \mathrm{~cm}$ above the umbilicus. All measurements will be in $\mathrm{cm}$, using an inelastic tape.

Individuals with a similar BMI may have different fat distributions. Thus, the waist-to-hip ratio (WHR) has been used as an additional measure of adiposity. ${ }^{32}$ The WHR and the waist-to-height ratio (WHtR) will be calculated by dividing the WC by the $\mathrm{HC}^{29}$ and by the height, respectively. ${ }^{33}$

A body shape index (ABSI) will be calculated as WC/ $\left(\mathrm{BMI}^{2 / 3} \times\right.$ height $\left.^{1 / 2}\right)$ using $\mathrm{WC}$ and height in metres, as suggested by Krakauer and Krakauer ${ }^{34}$ who determined that ABSI was a predictor of mortality. In addition, ABSI has been known to have a stronger association with total and cardiovascular mortality compared with other anthropometric measures in older adults. ${ }^{33}$

The heart and pericardial fat (which consists of epicardial and paracardial fat) will be washed in running water to remove clots, fixed in $70 \%$ alcohol for 1-14 days and weighed. Epicardial fat is located at the outer wall of the myocardium, and paracardial fat is located in the mediastinum outside of the parietal pericardium. ${ }^{15} 21$ Omental, mesenteric, mesocolon and perirenal fat will be dissected and weighed (figure 2). All visceral fat will be weighed using a calibrated electronic scale, and the measurements will be expressed in grams. Samples of visceral fat with dimensions of $0.5 \times 1.5 \mathrm{~cm}$ 
A

B

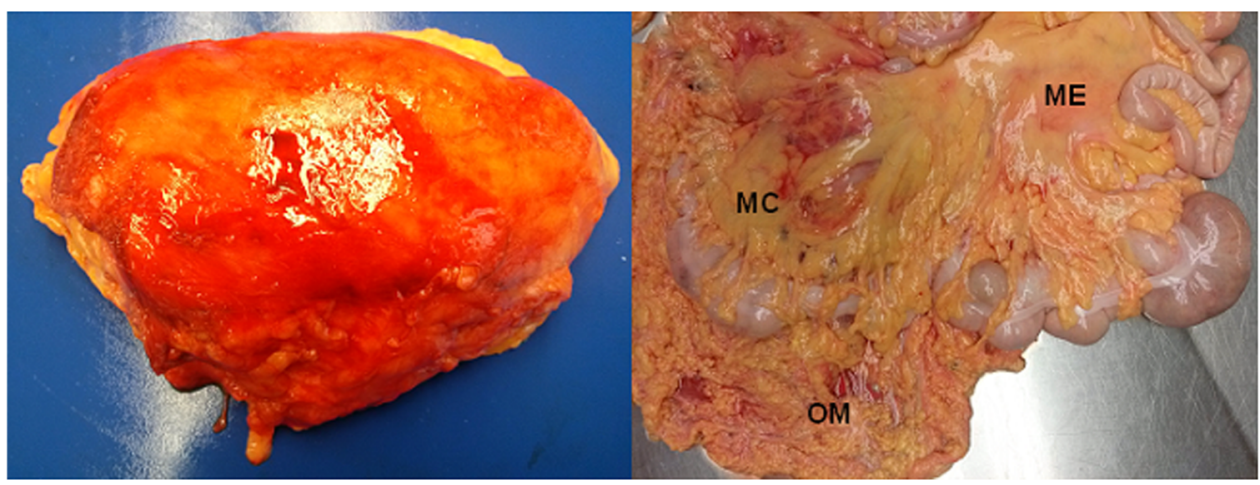

Figure 2 Abdominal visceral fat. (A) Perirenal fat attached to the kidney. (B) ME, OM and MC fat. ME, mesenteric; OM, omental; MC, mesocolon.

A

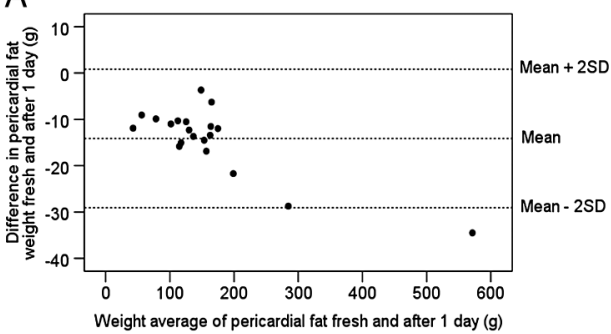

C

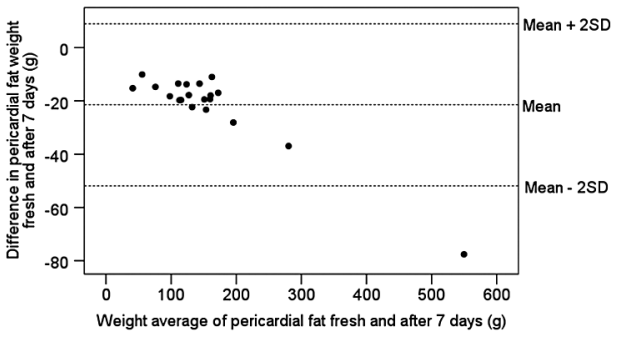

B

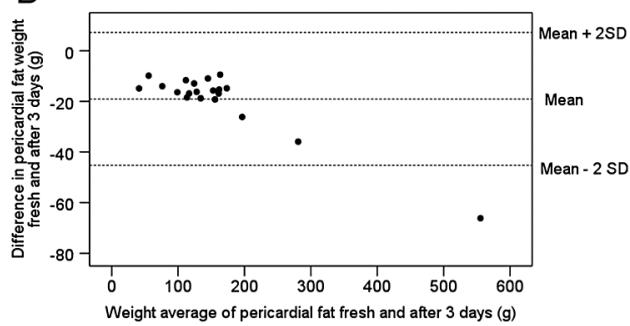

$\mathrm{D}$

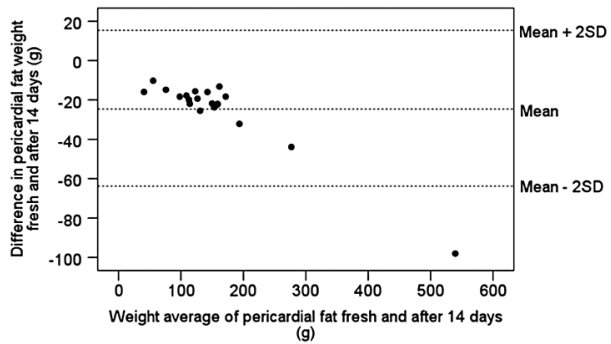

Figure 3 Plots of the difference between the weights of the fresh and fixed pericardial fat against their means. (A) Fresh weight and after 1 day of fixation; (B) fresh weight and after 3 days of fixation; (C) fresh weight and after 7 days of fixation and (D) fresh weight and after 14 days of fixation.

will be frozen in liquid nitrogen and stored in the freezer at $-80^{\circ} \mathrm{C}$, and other samples with dimensions of $1.5 \times 1.5 \mathrm{~cm}$ will be fixed in $10 \%$ formalin for future studies.

\section{Standardisation of pericardial fat fixation}

To evaluate the effect of different fixation times on pericardial fat weight, we collected pericardial fat from 20 individuals and we weighed the fat in natura and after fixation in $70 \%$ alcohol for $1,3,7$ and 14 days. Bland-Altman plots were used to analyse the agreement between the weights of the fresh and fixed pericardial fat. We plotted the difference between the weights of the fresh and fixed pericardial fat against their means, ${ }^{35}$ and it was within the agreement limits (figure 3 ). We also calculated the correlation between the weights of the fresh and fixed pericardial fat, and they were high for all the different times $(r>0.99)$.

\section{Evaluation of systemic atherosclerosis}

Carotid arteries will be dissected from the base of the aortic arch and stored in $70 \%$ alcohol for 24 hours. Gelatine will be injected inside the vessel lumen to prevent artery collapse, and the artery will be further fixed with $10 \%$ formalin. Subsequently, the common and internal carotid arteries will be cut transversely at $5 \mathrm{~mm}$ intervals, ${ }^{25}$ and five sections will be selected: (1) a section with the largest lumen obstruction of the common carotid artery, (2) the section of the common carotid artery located $1 \mathrm{~cm}$ below the carotid bifurcation, (3) the section located at the bifurcation, (4) the section of the internal carotid artery located $1 \mathrm{~cm}$ above the carotid bifurcation and (5) the section containing the largest lumen obstruction in the internal carotid artery. These cross-sections will be photographed with a stereomicroscope (Nikon SMZ 1000; Nikon Inst., New York, USA); the area will be delimited by the outer vessel wall, 


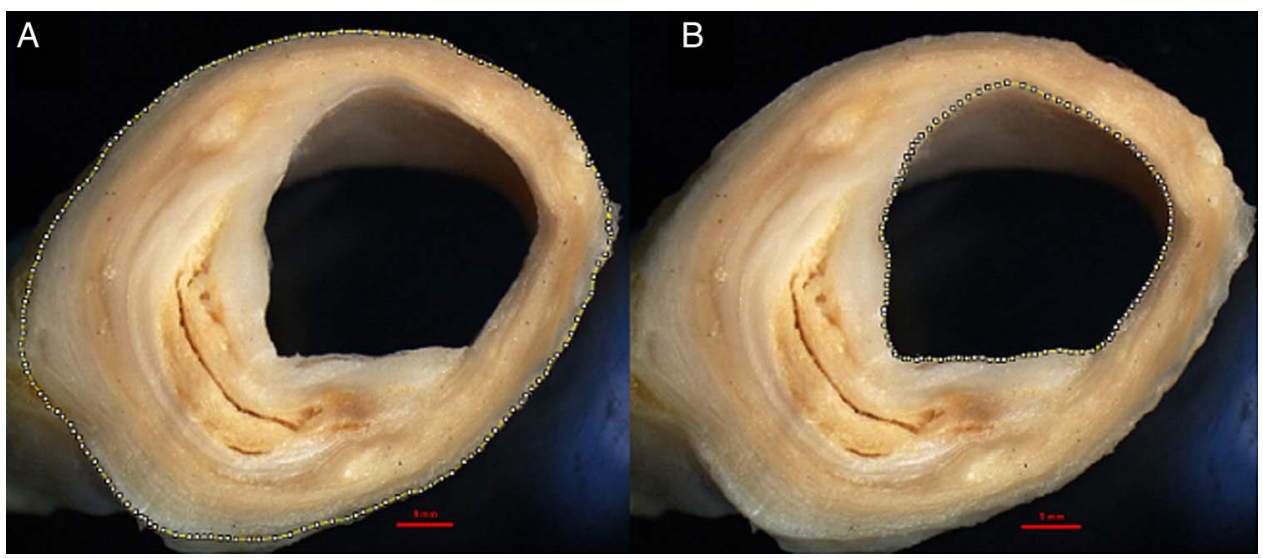

Figure 4 Measures used for the calculation of the carotid artery stenosis index. (A) Area limited by the outer wall of the vessel. (B) Lumen area.

and the lumen area will be measured using an image processor (Image J) (figure 4). For each segment, the stenosis index will be calculated by subtracting the lumen area from the outer area and dividing it by the outer area. The quotient will be then multiplied by $100 .^{36}$

The circle of Willis (CW) will be removed from the base of the brain and processed similarly to the carotid arteries. Subsequently, the following arteries of the CW will be cut into $3 \mathrm{~mm}$ thick cross-sections: the anterior cerebral arteries, the anterior communicating artery, the middle cerebral arteries, the internal carotid arteries (segments close to the CW), the posterior communicating arteries, the posterior cerebral arteries and the basilar artery. The segment with the largest lumen obstruction from each artery will be photographed using the stereomicroscope. The stenosis index will be calculated similarly to the carotid artery.

The left, circumflex, left anterior descending and right coronary arteries will be dissected and processed similarly to the carotid and CW arteries. They will be cut into $5 \mathrm{~mm}$ thick sections. The stenosis index of the largest plaque will be also calculated as previously described. The presence of myocardial infarction will be carefully evaluated by a certified pathologist. Lesions will be classified in acute or chronic myocardial infarcts according to the criteria of Kumar et al. ${ }^{37}$ Left ventricular wall thickness will be measured $1 \mathrm{~cm}$ below the mitral valve in $\mathrm{mm}$.

The number of atherosclerotic plaques in cerebral and coronary arteries will be counted to describe the extent of atherosclerotic disease.

We plan to evaluate atheroma plaque characteristics from the coronary, carotid and cerebral arteries. These samples will be submitted to the process of dehydration, diaphanisation and paraffin embedding. Then, the paraffin block will be cut into $4 \mu \mathrm{m}$ thickness sections using a microtome (Leica RM 2145; Leica Microsystems Brazil). The slides will be stained using H\&E and Verhoeff and photographed using a stereomicroscope. The evaluation of the plaque composition will be

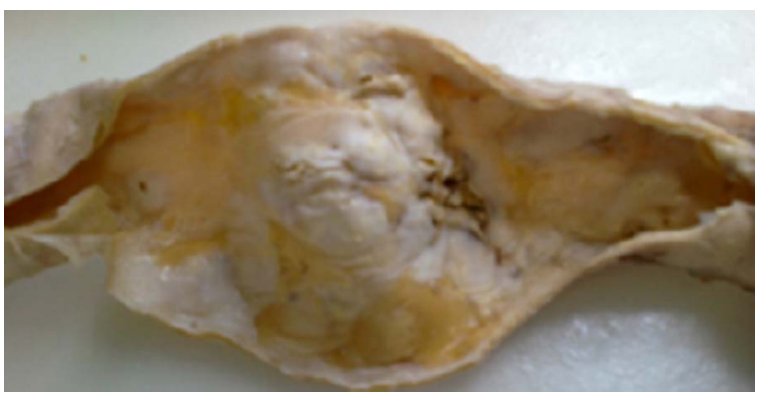

Figure 5 Evaluation of the aorta. Grade 3 of atherosclerosis with ulceration and multifocal protrusion.

performed according to the American Heart Association criteria and classified in types I-VIII. ${ }^{38}$

The aorta (ascending, thoracic and abdominal) will be opened longitudinally. The degree of atherosclerosis and the presence of confluent lesions (those that extend around the circumference of the aorta) will be investigated. Plaques will be classified as grade 1 if the plaques are not confluent and there is no ulcerations and protrusions, grade 2 if there are confluent areas or/ and an area of ulceration with minimal protrusion and grade 3 if there are confluent plaques, multifocal ulcerations or protrusions ${ }^{39}$ (figure 5).

\section{Sample size calculation}

The sample size was calculated on the basis of previous studies using the lowest correlation coefficient found in the literature from the study of Kortelainen and Särkioja ${ }^{16}$ ( $\mathrm{r}=0.109$ for the correlation of WC and perirenal fat). Assuming a power of $90 \%$ and $\alpha=0.05$, in a twotailed test, we will need a sample of 205 individuals. To better study different age groups, we will include at least 240 individuals in our sample.

\section{DATA ANALYSIS}

To describe the sample, we will use mean and SD or median and IQR for continuous variables and absolute 
and relative frequencies for categorical variables. If the distribution is Gaussian, an unpaired t-test will be used to compare the anthropometric measures (BMI, NC, WC, HC, WHR, WHtR, ABSI and ASTT) and the weight of visceral fat (thoracic, abdominal and total) between genders; otherwise we will use the Mann-Whitney test. We will use one-way ANOVA to compare these measurements among age groups $(30-49 ; 50-69 ; 70-89$ and 90 years or more) and types of myocardial infarction (control, acute, and chronic) if the distribution is Gaussian, otherwise we will use the Kruskal-Wallis test. We will use Tukey's HSD (honest significant difference) test in post hoc analyses to determine which mean value is significantly different among age groups and types of myocardial infarction.

We will use Pearson's correlation test (for continuous variables with Gaussian distribution) or Spearman's rank correlation coefficient (for continuous distribution with non-Gaussian distributed or ordinal variables) to compute the correlation of:

A. Weight of visceral fat with stenosis index in coronary, cerebral and carotid arteries.

B. Weight of visceral fat with degree of atherosclerosis in aorta.

C. Weight of visceral fat with number of plaques in coronary and cerebral arteries.

D. Anthropometric measurements with stenosis index in coronary, cerebral and carotid arteries.

E. Anthropometric measurements with degree of atherosclerosis in aorta.

F. Anthropometric measurements with number of plaques in coronary and cerebral arteries.

G. Weight of the heart and left ventricular wall thickness with the stenosis index in coronary arteries.

$\mathrm{H}$. Weight of the heart and left ventricular wall thickness with number of plaques in coronary arteries.

Linear regression analysis will be used to assess the association of visceral fat, anthropometric measurements, sociodemographic data and cardiovascular risk factors with atherosclerosis in coronary, carotid and cerebral arteries and will use ordered logistic regression analysis to assess the association of visceral fat, anthropometric measurements, sociodemographic data and cardiovascular risk factors with the degree of atherosclerosis in aorta. Results will be adjusted for age, gender, schooling, smoking and physical inactivity; and the weight of visceral fat will be adjusted for height. The significance level will be set at 0.05 in two-tailed tests. All analyses will be performed using the Stata/MP V.14 (StataCorp LP, College Station, Texas, USA).

\section{STRENGTHS AND LIMITATIONS OF THE STUDY}

The main limitation of this study is the absence of clinical follow-up while the participant was alive. However, previous studies performed by our group demonstrated good reliability of our interview compared with a gold-standard outpatient evaluation. ${ }^{40}$ In addition, the main variables in this study (eg, atherosclerosis, visceral fat and anthropometric measurements) will be directly measured and will not be affected by a lack of follow-up during the life of the participant. Another limitation is the convenience sample, since SPAS performs only autopsies on individuals who died of unknown natural causes of death; therefore, the sample will not be representative of all deaths in the city of Sao Paulo.

On the other hand, the structure provided by SPAS will allow us to get a large sample due to acceptance of NOK regarding tissue donation and participation in the study, good conditions of the deceased's tissue and reliable information provided by the NOK. Furthermore, this study has several strengths. The most important one will be the large number of participants. In comparison, most previous autopsy studies evaluated a lower number of cases, ranging from 30 to 50 individuals, ${ }^{4}$ 16-19 which is probably due to the decreasing number of autopsies performed worldwide. ${ }^{42}$ Another strength is the evaluation of older individuals while previous studies included a larger percentage of younger patients (Kortelainen and Särkioja: ${ }^{16}$ mean age 41 years (range: 19-63); Kortelainen and Särkioja: ${ }^{4} 35$ years (range: 19 49) and Kortelainen and Särkioja: ${ }^{18} 17$ years (range: 13 19)). The short PMI mean of 10.4 hours (4-20 hours) of the material collected by the BB-BABSG team ${ }^{24}$ allows for high-quality material for future genetic and proteomic studies. Moreover, our sample will be ethnically diverse, unlike previous autopsy studies for which the samples were mostly Caucasian. ${ }^{46-18}$ The present study also will perform morphometric measures of atherosclerotic plaques and objective measurements of visceral fat that are the gold standard compared with other clinical and imaging techniques. ${ }^{31}$ In fact, Kortelainen and Särkioja ${ }^{18}$ reported that the autopsy studies have the advantage of being able to measure the whole deposit of visceral fat and allows for complete assessment of the coronary tree. Additionally, our study will evaluate atherosclerosis not only in coronary arteries but also in the aorta, cerebral and carotid arteries, which is a unique aspect compared with other studies. Furthermore, most of the previous autopsy studies evaluated only abdominal visceral fat, while we also will evaluate pericardial fat.

Finally, we will use a unique autopsy service that already supported a variety of pathological projects to gather a high-quality and diverse tissue collection of arteries and visceral fat. This service also provides a comprehensive postmortem clinical evaluation. A large number of samples can be collected due to the great number of autopsies performed in our service. The stored fat and arterial samples will be an outstanding opportunity for future collaborative studies that will advance the understanding of the relationship between atherosclerosis pathophysiology and adiposity.

\section{DISSEMINATION}

After being informed about this study, the NOK of the deceased will sign a written informed consent. Results 
will be submitted for publication in a peer-reviewed journal and also presented at national and international conferences.

\section{Author affiliations}

${ }^{1}$ Laboratory of Cardiovascular Pathology (LIM-22), Department of Pathology, University of Sao Paulo Medical School, Sao Paulo, Brazil

2Pathophysiology in Aging Lab/Brazilian Aging Brain Study Group (LIM-22), University of Sao Paulo Medical School, Sao Paulo, Brazil

${ }^{3}$ Discipline of Geriatrics, University of Sao Paulo Medical School, Sao Paulo, Brazil

${ }^{4}$ University of São Paulo School of Nursing, Sao Paulo, Brazil

${ }^{5}$ Department of Neurology, Memory and Aging Center, University of California, San Francisco, California, USA

${ }^{6}$ Department of Pathology, University of Sao Paulo Medical School, Sao Paulo, Brazil

Contributors The International Committee of Medical Journal Editors (ICMJE) criteria for authorship have been met. AN, CKS and CAP designed the study. AN, DSF, FMC, KCSS and AC were responsible for collecting and processing data. CKS, REPL, RELF, LTG, JMF, WJF and CAP were responsible for general supervision of the research group and support in acquisition and analysis of data. AN drafted the manuscript. AN and CKS performed the statistical analysis. All authors read, revised the manuscript critically, making significant improvements, and approved the final version to be published.

Funding AN is supported by a scholarship from the CAPES (CAPES Foundation, Ministry of Education of Brazil). DSF is supported by a scholarship from the FAPESP (Sao Paulo Research Foundation, 2013/ 12290-3), and AC was supported by a scholarship from the FAPESP (12/ 25337-5).

Competing interests None declared.

Patient consent Obtained.

Ethics approval The study protocol was approved by the Ethics Committee of University of Sao Paulo Medical School (412/11), Sao Paulo, Brazil.

Provenance and peer review Not commissioned; externally peer reviewed.

Open Access This is an Open Access article distributed in accordance with the Creative Commons Attribution Non Commercial (CC BY-NC 4.0) license, which permits others to distribute, remix, adapt, build upon this work noncommercially, and license their derivative works on different terms, provided the original work is properly cited and the use is non-commercial. See: http:// creativecommons.org/licenses/by-nc/4.0/

\section{REFERENCES}

1. World Health Organization. Department of Measurement and Health Information. 2012. http://www.who.int/healthinfo/global burden disease/metrics_daly/en/ (accessed 8 Mar 2016).

2. Global Burden of Disease 2013 Mortality and Causes of Death Collaborators. Global, regional, and national age-sex specific all-cause and cause-specific mortality for 240 causes of death, 1990-2013: a systematic analysis for the Global Burden of Disease Study 2013. Lancet 2015;385:117-71.

3. Thapar A, Jenkins IH, Mehta A, et al. Diagnosis and management of carotid atherosclerosis. BMJ 2013;346:f1485.

4. Kortelainen ML, Särkioja T. Extent and composition of coronary lesions in relation to fat distribution in women younger than 50 years of age. Arterioscler Thromb Vasc Biol 1999;19:695-9.

5. Mahabadi AA, Massaro JM, Rosito GA, et al. Association of pericardial fat, intrathoracic fat, and visceral abdominal fat with cardiovascular disease burden: the Framingham Heart Study. Eur Heart J 2009;30:850-6.

6. Hassan M, Latif N, Yacoub M. Adipose tissue: friend or foe? Nat Rev Cardiol 2012;9:689-702.

7. Jeong JW, Jeong $\mathrm{MH}$, Yun $\mathrm{KH}$, et al. Echocardiographic epicardial fat thickness and coronary artery disease. Circ $J$ 2007;71:536-9.

8. Kim SK, Park SW, Kim SH, et al. Visceral fat amount is associated with carotid atherosclerosis even in type 2 diabetic men with a normal waist circumference. Int J Obes (Lond) 2009;33:131-5.
9. Taguchi R, Takasu J, Itani $\mathrm{Y}$, et al. Pericardial fat accumulation in men as a risk factor for coronary artery disease. Atherosclerosis 2001;157:203-9.

10. Ding J, Hsu FC, Harris TB, et al. The association of pericardial fat with incident coronary heart disease: the Multi-Ethnic Study of Atherosclerosis (MESA). Am J Clin Nutr 2009;90:499-504.

11. Natale F, Tedesco MA, Mocerino R, et al. Visceral adiposity and arterial stiffness: echocardiographic epicardial fat thickness reflects, better than waist circumference, carotid arterial stiffness in a large population of hypertensives. Eur $J$ Echocardiogr 2009;10:549-55.

12. Srinivasan $S R$, Wang $R$, Chen $W$, et al. Utility of waist-to-height ratio in detecting central obesity and related adverse cardiovascular risk profile among normal weight younger adults (from the Bogalusa Heart Study). Am J Cardiol 2009;104:721-4.

13. Alexopoulos N, Katritsis D, Raggi P. Visceral adipose tissue as a source of inflammation and promoter of atherosclerosis. Atherosclerosis 2014;233:104-12.

14. Schlett CL, Massaro JM, Lehman SJ, et al. Novel measurements of periaortic adipose tissue in comparison to anthropometric measures of obesity, and abdominal adipose tissue. Int $J$ Obes (Lond) 2009;33:226-32.

15. Bertaso AG, Bertol D, Duncan BB, et al. Epicardial fat: definition, measurements and systematic review of main outcomes. Arq Bras Cardiol 2013;101:e18-28.

16. Kortelainen ML, Särkioja T. Coronary atherosclerosis and myocardia hypertrophy in relation to body fat distribution in healthy women: an autopsy study on 33 violent deaths. Int $J$ Obes Relat Metab Disord 1997;21:43-9.

17. Kortelainen ML, Särkioja T. Extent and composition of coronary lesions and degree of cardiac hypertrophy in relation to abdominal fatness in men under 40 years of age. Arterioscler Thromb Vasc Biol 1997;17:574-9.

18. Kortelainen ML, Särkioja T. Visceral fat and coronary pathology in male adolescents. Int J Obes Relat Metab Disord 2001;25:228-32.

19. Rastogi P, Pinto DS, Pai MR, et al. An autopsy study of coronary atherosclerosis and its relation to anthropometric measurements/ indices of overweight and obesity in men. J Forensic Leg Med 2012;19:12-7.

20. Silaghi A, Piercecchi-Marti MD, Grino M, et al. Epicardial adipose tissue extent: relationship with age, body fat distribution, and coronaropathy. Obesity (Silver Spring) 2008;16:2424-30.

21. Sequeira DI, Ebert LC, Flach PM, et al. The correlation of epicardial adipose tissue on postmortem CT with coronary artery stenosis as determined by autopsy. Forensic Sci Med Pathol 2015;11:186-92.

22. Nakashima $Y$, Kiyohara $Y$, Doi $Y$, et al. Risk factors for coronary atherosclerosis in a general Japanese population: the Hisayama study. Pathol Res Pract 2009;205:700-8.

23. SVOC: Serviço de Verificação de Óbitos da Capital. http://www. svoc.usp.br (accessed 04 Apr 2015).

24. Grinberg LT, Ferretti RE, Farfel JM, et al. Brain bank of the Brazilian Aging Brain Study Group-a milestone reached and more than 1,600 collected brains. Cell Tissue Bank 2007;8:151-62.

25. Suemoto CK, Nitrini R, Grinberg LT, et al. Atherosclerosis and dementia: a cross-sectional study with pathological analysis of the carotid arteries. Stroke 2011;42:3614-5.

26. Farfel JM, Nitrini R, Suemoto CK, et al. Very low levels of education and cognitive reserve: a clinicopathologic study. Neurology 2013;81:650-7.

27. Crossley RP. Crossley Checklist-a system for determination of the time of death. Police Chief 1974;41(3):65-68, 85.

28. ABEP: Associação Brasileira de Empresas de Pesquisa. 2013. http://www.abep.org (accessed 07 Jul 2015).

29. World Health Organization. Waist Circumference and Waist-Hip Ratio: Report of a WHO Expert Consultation. Geneva: World Health Organization, 2008.

30. Laakso M, Matilainen V, Keinänen-Kiukaanniemi S. Association of neck circumference with insulin resistance-related factors. Int $J$ Obes Relat Metab Disord 2002;26:873-5.

31. van der Kooy K, Seidell JC. Techniques for the measurement of visceral fat: a practical guide. Int $J$ Obes Relat Metab Disord 1993;17:187-96.

32. Adabag S, Huxley RR, Lopez FL, et al. Obesity related risk of sudden cardiac death in the atherosclerosis risk in communities study. Heart 2015;101:215-21.

33. Dhana K, Kavousi M, Ikram MA, et al. Body shape index in comparison with other anthropometric measures in prediction of total and cause-specific mortality. J Epidemiol Community Health 2016;70:90-6.

34. Krakauer NY, Krakauer JC. Dynamic association of mortality hazard with body shape. PLoS One 2014;9:e88793. 
35. Bland JM, Altman DG. Statistical methods for assessing agreement between two methods of clinical measurement. Lancet 1986;1:307-10.

36. Roher AE, Esh C, Kokjohn TA, et al. Circle of Willis atherosclerosis is a risk factor for sporadic Alzheimer's disease. Arterioscler Thromb Vasc Biol 2003;23:2055-62.

37. Kumar V, Abbas AK, Fausto N, et al. Robbins and Cotran: pathologic basis of disease. 8th edn. Philadelphia: Elsevier Saunders, 2010.

38. Stary HC. Natural history and histological classification of atherosclerotic lesions: an update. Arterioscler Thromb Vasc Biol 2000;20:1177-8.
39. Dolan $\mathrm{H}$, Crain $\mathrm{B}$, Troncoso J, et al. Atherosclerosis, dementia, and Alzheimer disease in the Baltimore Longitudinal Study of Aging cohort. Ann Neurol 2010;68:231-40.

40. Ferretti REL, Damin AE, Brucki SMD, et al. Post-mortem diagnosis of dementia by informant interview. Dement Neuropsychol 2010;4:138-44.

41. Apolinario D, Brucki SM, Ferretti RE, et al. Estimating premorbid cognitive abilities in low-educated populations. PLOS One 2013;8: e60084.

42. Burton JL, Underwood J. Clinical, educational, and epidemiological value of autopsy. Lancet 2007;369:1471-80. 